\title{
REVIEW
}

\section{Design and Implementation of Automatic Following Balancing Vehicle Based on UWB Positioning Principle}

\section{Chenyu Zhang* Fangyi Chen Zhongyun Kang}

Information Department, Beijing University of Technology, Beijing, 100124, China

\begin{tabular}{l}
\hline ARTICLE INFO \\
\hline Article history \\
Received: 27 February 2020 \\
Revised: 5 March 2020 \\
Accepted: 9 April 2020 \\
Published Online: 16 April 2020 \\
\hline
\end{tabular}

Keywords:

Automatic following trolley

UWB positioning

TOF ranging principle

\begin{abstract}
With the modernization of industrial production, various automatic following technologies have gradually penetrated into people's lives. Among them, the application fields that follow mobile balancing vehicles range from the structured environment of factories to the daily living environment. The purpose of this subject is to be able to use UWB positioning technology to realize that the balanced trolley is an automatic following function, which mainly uses the ranging function in UWB positioning technology, uses the TOF ranging principle and embedded basic knowledge, and changes the positioning algorithm to a following algorithm. The distance between the target object and the person is effectively determined by two tags set on the trolley and a base station in the hand to determine the distance, and the distance information is transmitted through the serial port. By writing an algorithm, the distance information is converted to the driving information of the trolley, and the driving information is sent to the trolley to control the trolley, thereby realizing the task of balancing the trolley to follow the specified target in a simple indoor environment.
\end{abstract}

detection sensors for unmanned vehicles. Commonly used communication technologies include infrared, ultrasonic, radio frequency signals, computer binocular stereo vision sensing, etc., but they are not suitable for indoor signal transmission. Infrared is only suitable for short-distance transmission, and is easily interfered by fluorescent lights or lights in the room. Mobile robots relying on ultrasonic or infrared ranging sensors to avoid obstacles have limited detection ranges and insufficient data at detection points, these defects affect the accuracy of obstacle avoidance control of unmanned vehicles; ultrasonic waves are greatly affected by multipath effects and non-line-of-sight

\footnotetext{
*Corresponding Author:

Chenyu Zhang,

female, undergraduate of Beijing University of Technology;

Correspondence address: Room 202, Unit 3, Building 4, District 2, Guoling Town, Changping District, Beijing, China;

E-mail:1648215006@qq.com.
} 
propagation and cannot be used in indoor environments; radio frequency signals are commonly used in outdoor transmission systems, but they have limitations in indoor systems. Monocular vision can only obtain two-dimensional image information of the environment and cannot obtain depth information of environmental obstacles. Binocular vision has disadvantages such as poor real-time performance and complicated calculation.

It can be seen that with the development of wireless network technology and the increasing demand for follow-up services, the automatic follow-up technology must overcome the shortcomings of the existing technology and meet the following conditions: a) High anti-interference ability; b) High accuracy; c) Low production cost; d) Low operating cost; e) High information security; f) Low energy consumption and low transmit power; g) Small transceiver volume.

None of the above technical solutions can fully meet these requirements. UWB is used for auto-following, which can basically meet the above requirements. UWB is a highspeed, low-cost and low-power emerging wireless communication technology. UWB signals are pulse signals with a bandwidth greater than $500 \mathrm{MHz}$ or a ratio of the baseband bandwidth to the trolleyrier frequency greater than 0.2 (UWBWG, 2001), and have a wide frequency band range. It can be seen that UWB focuses on applications in two fields. On the one hand, it is a short-range high-speed data communication that complies with the IEEE802.15.3a standard, that is, wirelessly transmits a large amount of multimedia data without delay, and the rate must reach $100 \mathrm{Mbit} / \mathrm{s}-500 \mathrm{Mbit} / \mathrm{s}$; on the other hand, it is low-speed and low-power transmission in accordance with IEEE802.15.4a, which is used for accurate indoor positioning, such as position detection of battlefield soldiers, industrial automation, sensor networks, home / office automation, robot motion tracking, and so on. The characteristics of UWB signals indicate that it has the advantages of low cost, anti-multipath interference, and strong penetrability in communication, so it can be applied to the positioning and tracking of stationary or moving objects and people, and can provide very accurate positioning accuracy ${ }^{[1,5,7]}$

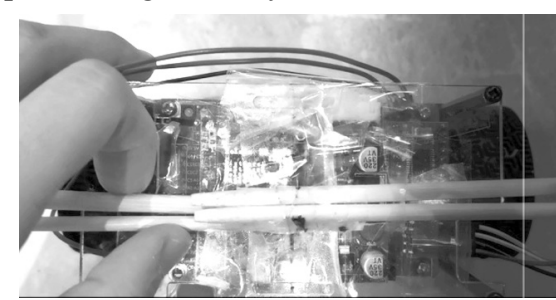

Figure 1. Trolley display screen physical diagram

\section{UWB Ranging Module}

The TOF development board is based on the official orig- inal Evaluation board for new PCB upgrade and development. Based on the EVB, the LCD display and redundant interfaces are deleted to optimize power supply design and routing. The module also uses the STM32F 105 microcontroller as the main control chip, which inherits and optimizes the official embedded code to the greatest extent. Peripheral circuits include: DWM1000 module (DWSMA module $+40 / \mathrm{pcs}$ ), power module, LED indicator module, DIP switch, etc. The development board can be used as a base station or a tag, and can be switched by a DIP switch.

\subsection{Main Control Chip: STM32 F105}

STM32F105 is an interconnected 32-bit ARM-based microcontroller with 64 or $256 \mathrm{~K}$ bytes of flash memory. It has USB OTG, Ethernet, 10 timers, 2 CAN, 2 ADCs, 14 communication interfaces. ${ }^{[8]}$

\subsection{Communication Chip: DW1000}

The DWM1000 module is an ultra-wideband transceiver module designed based on the DW1000 chip of DECAWAVE. This module integrates the antenna and all RF circuits, power management and clock circuits. This module can be used in TWR or TDOA positioning systems to locate targets with an accuracy of less than 10CM; and the module supports data transmission rates up to $6.8 \mathrm{MBPS} .^{[12]}$

\subsection{Ranging Algorithm}

TOF (Time of Flight): The ranging method is a twoway ranging technology. It mainly uses the time of flight between two asynchronous transceivers (Transceiver) to measure the distance between nodes. In the line-of-sight environment, the TOF-based distance measurement method has a linear relationship with distance, so the results will be more accurate. We record the time between the data packet sent by the sender and the response as TTOT, and the time interval between the time the data packet is received and the response sent by the receiver is TTAT, then the time TTOF of the unidirectional flight of the data packet in the air can be calculated as:

TTOF $=($ TTOT-TTAT $) / 2$

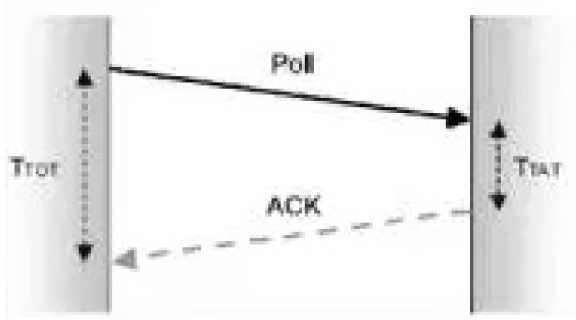

Figure 2. Ranging algorithm schematic diagram 
But the simple TOF algorithm has a stricter constraint: the sending device and the receiving device must always be synchronized

TW-TOF (two-way time of flight method):

(a) Unilateral two-way ranging

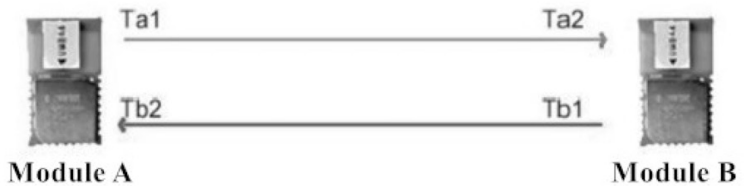

Figure 3. Unilateral two-way ranging algorithm schematic diagram 1

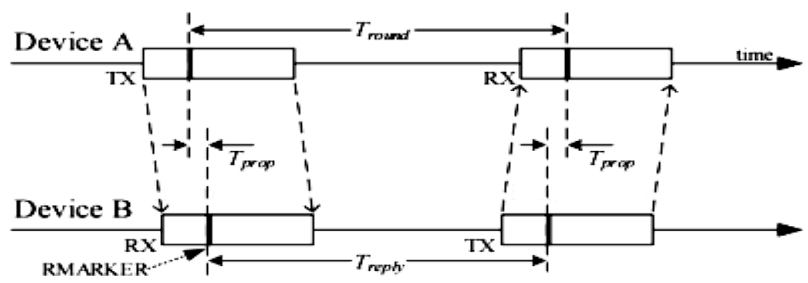

Figure 4. Unilateral two-way ranging algorithm schematic diagram 2

Device A first sends a data packet to device B, and records the time of sending the packet Ta1. After receiving the data packet, device $\mathrm{B}$ records the time of receiving the packet Tb1. After that, device B waits for the Treply moment and sends a data packet to device $\mathrm{A}$ at the time $\mathrm{Tb} 2$ $(\mathrm{Tb} 2=\mathrm{Tb} 1+$ Treply). After receiving the data packet, device A records the time value Ta2. Then you can calculate the time Tprop of electromagnetic waves in the air, multiplying the time of flight by the speed of light is the distance between the two devices.

Because device A and device B use separate clock sources, the clocks will have a certain deviation. Assuming that the actual frequency of the clocks of device $A$ and device $\mathrm{B}$ is eA and eB times the expected frequency, then the error introduced by the clock deviation is: error $=$ Tprop $\times(1-(k a+k b) / 2)$.

(b) Bilateral two-way ranging

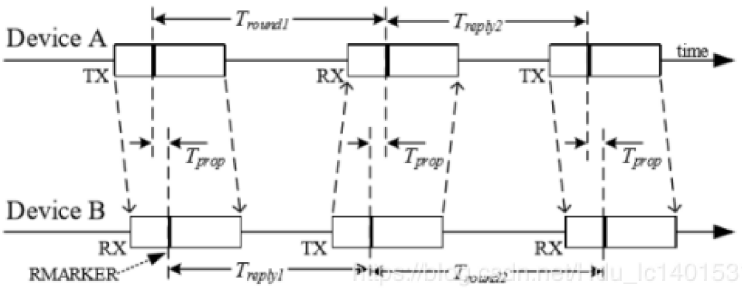

Figure 5. Bilateral two-way ranging algorithm schematic diagram

$$
\widehat{T}_{\text {prop }}=\frac{\left(T_{\text {round } 1} \times T_{\text {round } 2}-T_{\text {reply } 1} \times T_{\text {reply } 2}\right)}{\left(T_{\text {round } 1}+T_{\text {round } 2}+T_{\text {reply } 1}+T_{\text {reply } 2}\right)}
$$

DS ranging is based on SS ranging, and one more communication is added. The two communication times can compensate each other for errors introduced by clock skew.

The error introduced by the DS ranging method clock is: error $=$ Tprop $\times(1-(\mathrm{ka}+\mathrm{kb}) / 2)$.

Suppose the clock accuracy of device A and device B is $20 \mathrm{ppm}$ (very poor), and $1 \mathrm{ppm}$ is one millionth. Then $\mathrm{Ka}$ and $\mathrm{Kb}$ are 0.99998 or 1.00002 respectively, and ka and $\mathrm{kb}$ are the ratios of the actual frequency and the expected frequency of the clocks of the devices A and B, respectively. The distance between equipment $\mathrm{A}$ and $\mathrm{B}$ is $100 \mathrm{~m}$, and the flight time of electromagnetic waves is $333 \mathrm{~ns}$. Then the error introduced by the clock is $20 * 333$ * 10-9 seconds, the ranging error is $2.2 \mathrm{~mm}$, which can be ignored. ${ }^{[4]}$

\section{Trolley Hardware Module}

\subsection{Main Control Module}

The STM32F103xC, STM32F103xD and STM32F103xE enhanced series use the high-performance ARM ${ }^{\circledR}$ Cortex TM -M3 32-bit RISC core and operate at $72 \mathrm{MHz}$, built-in high-speed memory (up to $512 \mathrm{~K}$ bytes of flash and $64 \mathrm{~K}$ bytes of SRAM), rich enhanced I / O ports and peripherals connected to two APB buses. All devices include three 12-bit ADCs, four general-purpose 16-bit timers, and two PWM timers. They also include standard and advanced communication interfaces: up to 2 I2C interfaces, 3 SPI interfaces, 2 I2S interfaces, 1 SDIO interface, 5 USART interfaces, a USB interface and a CAN interface. The STM32F103xx high-capacity enhanced series operates in the temperature range of $-40{ }^{\circ} \mathrm{C}$ to $+105^{\circ} \mathrm{C}$, and the power supply voltage is $2.0 \mathrm{~V}$ to $3.6 \mathrm{~V}$. A series of power saving modes guarantee the requirements of low power consumption applications. STM32F103xx high-capacity enhanced series products provide 6 different package forms from 64 pins to 144 pins; the configuration of the peripherals in the device varies depending on the package. Below is a basic introduction to all the peripherals in this series of products. ${ }^{[1]}$

\subsection{Motor Drive Module}

TB6612FNG is a driver chip for DC motors, whose output transistor uses a low-resistance LD-MOS structure. The two input signals IN1 and IN2 can select one of four modes, such as $\mathrm{CW}, \mathrm{CCW}$, short brake and stop modes. ${ }^{[10]}$ 


\subsection{Balance Module}

MPU6000 (6050) is the world's first integrated 6-axis motion processing component. Compared with the multi-component solution, it eliminates the problem of the difference between the time axis of the combined gyroscope and the accelerator and reduces a lot of packaging space. When connected to a three-axis magnetic timing, the MPU-60X0 provides a full 9-axis motion fusion output to its main I2C or SPI port (SPI is only available on the MPU-6000). ${ }^{[6]}$

\section{System Model and Algorithm}

The realization of the auto-following balance trolley based on the UWB positioning principle needs to implement data reception, conversion processing, control of the trolley according to distance information, etc. Take oneto-one communication as an example to build a system model and data processing flow:

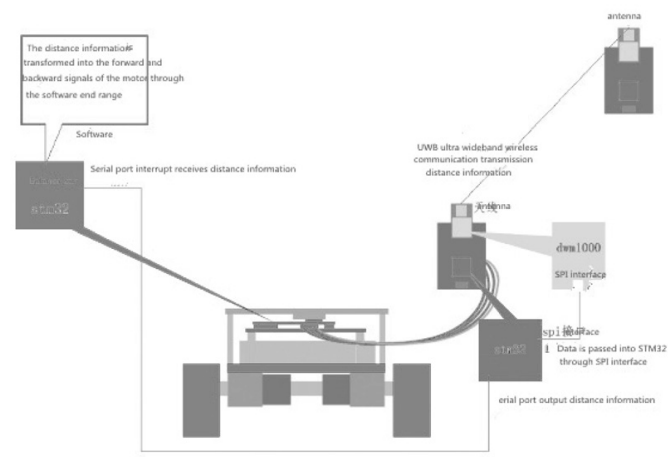

Figure 6. Communication principle between the trolley module and the ranging module

In order to realize the function of turning in the left and right of automatic following, the realization of the automatic following balancing trolley based on the UWB positioning principle requires three positioning modules.

Base station 0 is connected to serial port 2 of the trolley through a serial port, base station 1 is connected to serial port 4 of the trolley through a serial port, and a person holds a tag 0 to send and receive distance information data. The main control chip of the trolley stores two distance information and makes a judgment. First, determine the distance between the two, convert it into a left-turn and right-turn instruction, and then calculate the vertical distance through a mathematical formula. And the distance between the two base stations and the tags are displayed on the OLED screen of the trolley.

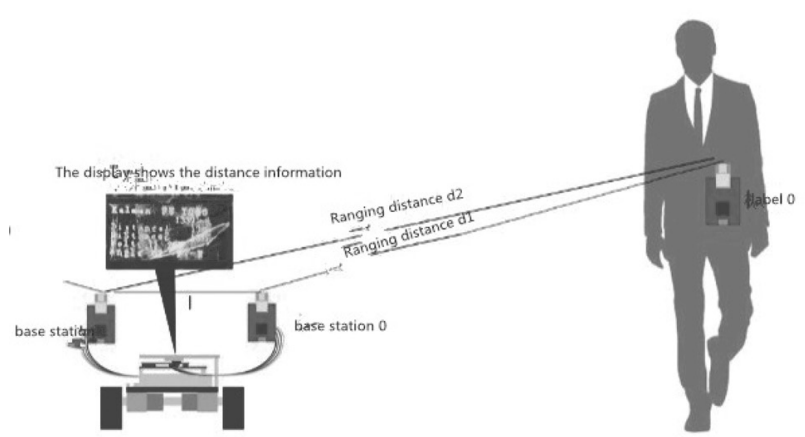

Figure 7. Trolley auto-following Schematic diagram

Vertical distance calculation algorithm:

(a) First compare the sizes of $\mathrm{d} 1$ and $\mathrm{d} 2$, and perform left and right turn control (until $\mathrm{d} 1>\mathrm{d} 2$ : turn right; $\mathrm{d} 1<\mathrm{d} 2$ turn left).

(b) Calculate the vertical distance through the mathematical formula of the triangle: Knowing the distance $\mathrm{L}$ between the base station 1 and the base station 2, the UWB ranging module actually measures the distance between each base station and the tag. The distance between base station 1 and label 0 is $\mathrm{d} 1$, and the distance between base station 0 and label 0 is $\mathrm{d} 2$.

Using the known three sides in the cosine theorem, first calculate the cosine of $\mathrm{C}$ :

$$
\cos C=\left(d \_1 \wedge 2+d \_2 \wedge 2-L_{-} \wedge 2\right) /\left(2 d \_1 d \_2\right)
$$

Then find the sine of $\mathrm{C}$ :

$$
\sin C=\sqrt{ }\left(1-(\cos C)^{\wedge} 2\right)
$$

From the properties of the triangle sine theorem, the area of the triangle is obtained:

$$
\mathrm{S}=0.5 \times \mathrm{d}_{-} 2 \times \sin \mathrm{C}
$$

From $0.5 \times \mathrm{L} \times \mathrm{d}$ is also the area of the triangle, which is obtained from the principle of equal area: $\mathrm{d}=\mathrm{S} /(0.5 \times$ $\sin C)$.

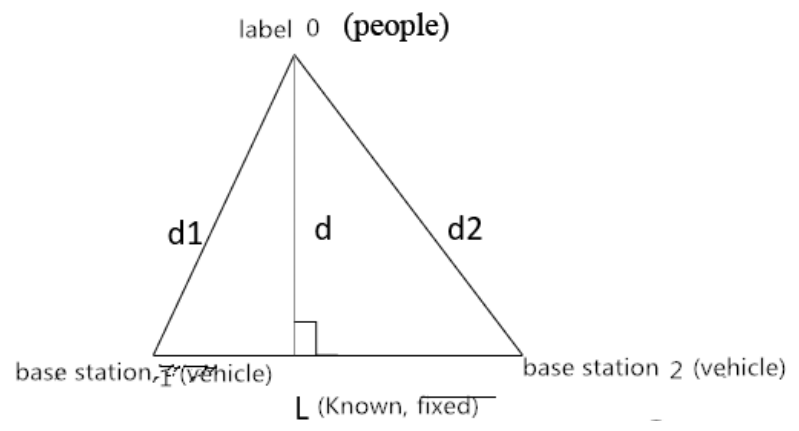

Figure 8. Trolley ranging algorithm schematic diagram 
The obtained vertical distance can be compared with the set distance. When the vertical distance is less than $1500 \mathrm{~mm}$, the trolley is controlled to advance, otherwise the trolley stops.

\section{Software Design}

After determining the idea through the theoretical model and algorithm, to realize the automatic following of the balance trolley, it is necessary to write the following algorithm as a program. We mainly use mcuisp software to download and update the trolley program through the serial port; use XCOM V2.0 software to test the DW1000 chip, realize serial communication data transmission, modify the transmission distance information format, and display the distance information on XCOM V2.0 serial debugging assistant software.

\section{Experimental Tests and Results}

The experimental location was set in a house with obstacles, and the ambient temperature was about $20{ }^{\circ} \mathrm{C}$. The final design of the trolley runs smoothly, and the corners are in good driving conditions. When the trolley is driven for a long time, the overall performance of the trolley is relatively stable, with occasional large deviations. As shown in Figure 9, three different follow-up route tests were performed on the trolley. The experimental results are listed in Table 1. In general, the trolley designed in this paper can realize the automatic follow target more accurately and smoothly. ${ }^{[2]}$

\section{Conclusion}

This paper designs an automatic following trolley based on the UWB positioning principle, and explains in detail the UWB module ranging principle, the hardware structure of the trolley, and the following algorithm. The trolley is suitable for indoor and outdoor obstacle environments, and can be modified to automatically follow the trunk and the factory automatically follow the small loading truck. There are certain defects in this trolley person. For example, when encountering obstacles such as large partitions, they cannot automatically detect and avoid; the base station cannot make correct judgments about the situation behind the balance trolley itself, and people need further experimental research to improve it.

\section{References}

[1] Liang Jiuying. Wireless positioning system[J]. Beijing: Electronic Industry Press, 2013:132-400.
Table 1. Test results of the following routes

\begin{tabular}{|c|c|c|c|c|}
\hline $\begin{array}{c}\text { Following } \\
\text { curve }\end{array}$ & $\begin{array}{c}\text { Number } \\
\text { of experi- } \\
\text { ments }\end{array}$ & $\begin{array}{c}\text { Number of } \\
\text { Successes }\end{array}$ & $\begin{array}{c}\text { Average } \\
\text { time }\end{array}$ & Success rate \\
\hline straight line & 6 & 6 & 35 & $100 \%$ \\
\hline $\begin{array}{c}\text { S-shape (turn } \\
\text { over 30 }\end{array}$ & 6 & 6 & 38 & $100 \%$ \\
\hline Round & 6 & 5 & 45 & $83.3 \%$ \\
\hline
\end{tabular}

Notes: All following lengths are $5000 \mathrm{~mm}$ and the following vertical distance is $1500 \mathrm{~mm} \cdot{ }^{[2]}$
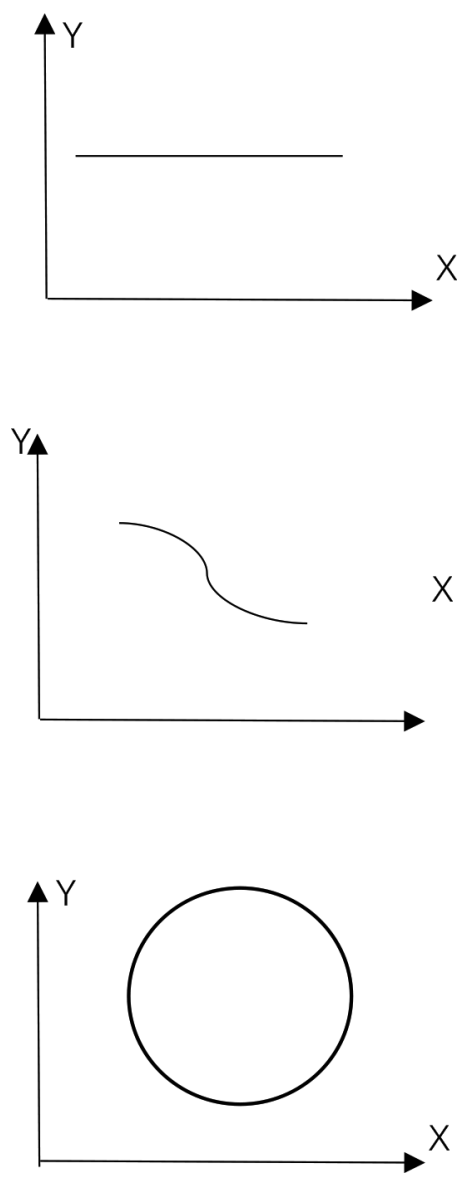

Figure 9. The experimental route schematic diagram

[2] Liu Jinhai, Wang Bofan, Zhou Long, Zheng Yuejiu, etc. Design of automatic following trolley based on UWB technology[D]. Shanghai: University of Shanghai for Science and Technology, 2019.

[3] Fang Chenchen. UWB-based autonomous following robot positioning method[D]. Shanghai: University of Shanghai for Science and Technology, 2016.

[4] Xiongda UWB. Xiongda UWB tutorial series[EB/ OL]. 2019-08-25.

[5] Andrew S. Tanenbaum. Computer network[J]. Beijing: Tsinghua University Press, 2012:85-89, 248- 
251.

[6] LeeDy.Li. MPU-6000 / MPU-6050 Product description[DB/OL]. 2013-01-07.

[7] Theodore S. Rappaport, Meng Qingming, etc. Principles and Applications of Wireless Communication (Second Edition) $[\mathrm{J}]$. Beijing: Electronic Industry Press, 2018:85-89, 120-122, 256-276.

[8] Liu Huoliang, Yang Sen, etc. Practical Guide for STM32 Library Function Development (Second Edition)[J]. Beijing: Machinery Industry Press, 2017:15-
20, 102-205.

[9] Zheng Fei. Application of TDOA-based CHAN algorithm in UWB and NLOS environments[D]. Guilin: University of Electronic Science and Technology of China, 2016.

[10] TOSHIBA. TB6612FNG[DB/OL]. 2012-11-01.

[11] STM32F103xCDE data manual English 5th edition.2009-3.

[12] DECACAVE.DW1000 USER MANUAL.2016. 